\title{
Hyaluronic gel injection into the vesicovaginal septum for high-dose-rate brachytherapy of uterine cervical cancer: an effective approach for bladder dose reduction
}

\author{
Naoya Murakami, MD, PhD', Satoshi Shima, MD!, Tairo Kashihara, MD! Nikolaos Tselis, MD, PhD², Tomoyasu Kato, MD, PhD², \\ Yoshiaki Takagawa, MD!, Koji Masui, MD, PhD', Ken Yoshida, MD, PhD ${ }^{5}$, Kana Takahashi, MD, PhD', Koji Inaba, MD, PhD!, \\ Kae Okuma, MD, PhD', Hiroshi lgaki, MD, PhD!', Yuko Nakayama, MD, PhD', Jun Itami, MD, PhD'
}

'Department of Radiation Oncology, National Cancer Center Hospital, Tokyo, Japan, ${ }^{2}$ Department of Radiotherapy and Oncology, GoetheUniversity Frankfurt, Germany, ${ }^{3}$ Department of Gynecologic Oncology, National Cancer Center Hospital, Tokyo, Japan, ${ }^{4}$ Department of Radiology, Kyoto Prefectural University of Medicine, Kyoto, Japan, ${ }^{5}$ Department of Radiation Oncology. Osaka Medical College, Takatsuki, Osaka, Japan

\begin{abstract}
Purpose: The purpose of this study was to report our initial experience of hyaluronic acid gel injection (HGI) in the vesicovaginal septum (VVS) for bladder dose reduction in brachytherapy (BT) for uterine cervical carcinoma.

Material and methods: Between September 2016 and May 2018, 15 uterine cervical cancer patients received HGI in the VVS as a part of their definitive radiotherapy (RT) treatment consisting of external beam radiation therapy (EBRT) with additional BT. Of those, 9 patients received BT both with and without HGI, and remaining 6 patients were excluded because these 6 patients received HGI in the VVS for all BT fractions. All 9 patients received HGI in the rectovaginal septum. For these patients, the dosimetric parameters bladder $\mathrm{D}_{2 c c^{\prime}}$ HR-CTV $\mathrm{D}_{90}$, and rectum $\mathrm{D}_{2 \mathrm{cc}}$ were selected, and two groups were generated (BT with vs. without HGI in the VVS) for dosimetric comparison.

Results: The median cumulative $\mathrm{EQD}_{2}$ for $\mathrm{HR}-\mathrm{CTV}$, rectum $\mathrm{D}_{2 \mathrm{cc}}$ and bladder $\mathrm{D}_{2 \mathrm{cc}}$ for the 9 patients were $73.3,52.8$, and 67.1, respectively. While no statistical difference could be detected for rectal dose reduction, bladder dose was significantly less in the group with HGI in the VVS compared to that without (449 cGy [range, 416-566, 1SD = 66.1] vs. 569 cGy [range, 449-647, $1 \mathrm{SD}=59.5], p=0.033$ ), with no compromising of target coverage. Although it did not reach statistically significance, there was a trend toward better HR-CTV $D_{90}$ in the group with HGI compared to that without HGI in the VVS (713 cGy vs. 706 cGy, $p=0.085$ ). No severe bleeding, hematuria, bladder wall injury, or urethral injury requiring hospitalization was experienced in association with HGI in the VVS.

Conclusions: HGI in the VVS can be performed safely and can effectively reduce the bladder dose in BT for uterine cervical cancer patients.

J Contemp Brachytherapy 2019; 11, 1: 1-7 DOI: https://doi.org/10.5114/jcb.2019.82612

Key words: uterine cervical cancer, brachytherapy, gel spacer injection, bladder dose, vesicovaginal septum.

\section{Purpose}

Brachytherapy (BT) plays an evidence-based role in the definitive radiotherapy (RT) of uterine cervical cancer $[1,2,3,4,5]$, with its impact being reflected in the direct correlation between its utilization and improvement in survival $[6,7]$. Furthermore, the introduction of image-guided adaptive brachytherapy (IGABT) facilitated the generation of clinical knowledge concerning the optimal dose coverage of the high-risk clinical target volume (HR-CTV), i.e.

over 85 Gy to achieve long-term local control as well as the dose constraints of adjacent organs at risk (OARs), such as rectum, sigmoid colon, and bladder $[8,9,10,11]$. Even though three-dimensional (3D) treatment planning in highdose-rate (HDR) IGABT enables for anatomy-oriented dose optimization, while the versatility of intratarget dose modulation can be controlled and directed to selectively reduce the dose to OARs, the anatomical relationship between tumor and adjacent risk structures does not always allow the compliance with dosimetric recommendations.
\end{abstract}


Table 1. Patients characteristics $(n=9)$

\begin{tabular}{lc} 
Median age & 69 (range, 35-87) \\
\hline FIGO stage I/II/III/IV & $0 / 8 / 1 / 0$ \\
\hline Pathology SqCC/Adeno & $9 / 0$ \\
$\begin{array}{l}\text { FIGO - International Federation of Gynecology and Obstetrics, SqCC - squa- } \\
\text { mous cell carcinoma, Adeno - adenocarcinoma }\end{array}$
\end{tabular}

Hyaluronic acid gel injection (HGI) has been shown to safely increase the space between OARs and target volumes in the BT treatment of various tumor sites, hence improving OARs protection significantly $[12,13,14,15]$. In addition, a phase III clinical trial could demonstrate that SpaceOAR ${ }^{\mathrm{TM}}$, the polymerized type of polyethylene-glycol hydrogel, effectively lowered the rectal dose in prostate cancer external beam radiation therapy (EBRT) $[16,17]$. The purpose of this study was to report our initial experience of HGI in the vesicovaginal septum (VVS) for patients treated with BT for uterine cervical cancer and to demonstrate its effectiveness with regards to bladder dose reduction.

\section{Material and methods}

Between September 2016 and May 2018, 15 uterine cervical cancer patients received HGI in the VVS for HDR-BT as a part of their definitive RT treatment. Of them, 9 patients received BT both with and without HGI in the VVS and were analyzed in this study. Remaining 6 patients were excluded because these 6 patients received HGI in the VVS for all BT fractions. All 9 patients also received HGI in the rectovaginal septum (RVS). Our RT management for uterine cervical cancer has been described in detail elsewhere $[18,19]$. In brief, whole pelvic EBRT of 20-40 Gy with sequential central shield (CS) of 10-30 Gy was delivered until a total pelvic dose of $50 \mathrm{~Gy}$ in 25 fractions. Concurrent weekly CDDP $\left(40 \mathrm{mg} / \mathrm{m}^{2}\right)$ was administered for patients with clinically positive
Table 2. Radiation therapy details $(n=9)$

\begin{tabular}{|c|c|}
\hline Median WPRT dose (Gy) & 30 (range, 20-50) \\
\hline Median CS dose (Gy) & 20 (range, 0-30) \\
\hline $\begin{array}{l}\text { Median HR-CTV } D_{90} \\
(G y, E Q D 2, \alpha / \beta=10 \text { Gy) }\end{array}$ & 73.3 (range, 59.0-82.3) \\
\hline $\begin{array}{l}\text { Median rectum } D_{2 c c} \\
(G y, E Q D 2, \alpha / \beta=3 \text { Gy) }\end{array}$ & 52.8 (range, 46.2-70.6) \\
\hline $\begin{array}{l}\text { Median bladder } D_{2 c c} \\
(G y, E Q D 2, \alpha / \beta=3 G y)\end{array}$ & 67.1 (range, 59.3-71.3) \\
\hline Number of BT sessions & 4 (range, 2-5) \\
\hline \multicolumn{2}{|l|}{ BT sessions with $\mathrm{HGI}$ in the VVS } \\
\hline Once & 4 \\
\hline 2 times & 1 \\
\hline 3 times & 3 \\
\hline 4 times & 1 \\
\hline \multicolumn{2}{|l|}{ Type of applicators used } \\
\hline Tandem \& ovoid & 2 \\
\hline Tandem \& ovoid \& needle & 6 \\
\hline Tandem \& cylinder & 1 \\
\hline Tandem \& cylinder \& needle & 0 \\
\hline
\end{tabular}

WPRT - whole pelvic radiation therapy, CS - central shield, HR-CTV - high-risk clinical target volume, BT - brachytherapy, HGI - hyaluronic acid gel injection, VVS - vesicovaginal septum

nodes or tumor size $>4 \mathrm{~cm}$. Brachytherapy was delivered after the initiation of CS by means of tandem and ovoids/ cylinder with or without additional interstitial needles for larger or irregular shaped tumors, according to the residual tumor extent at the time of BT. All 9 analyzed patients' characteristics are presented in Table 1 with Table 2 summarizing RT details.
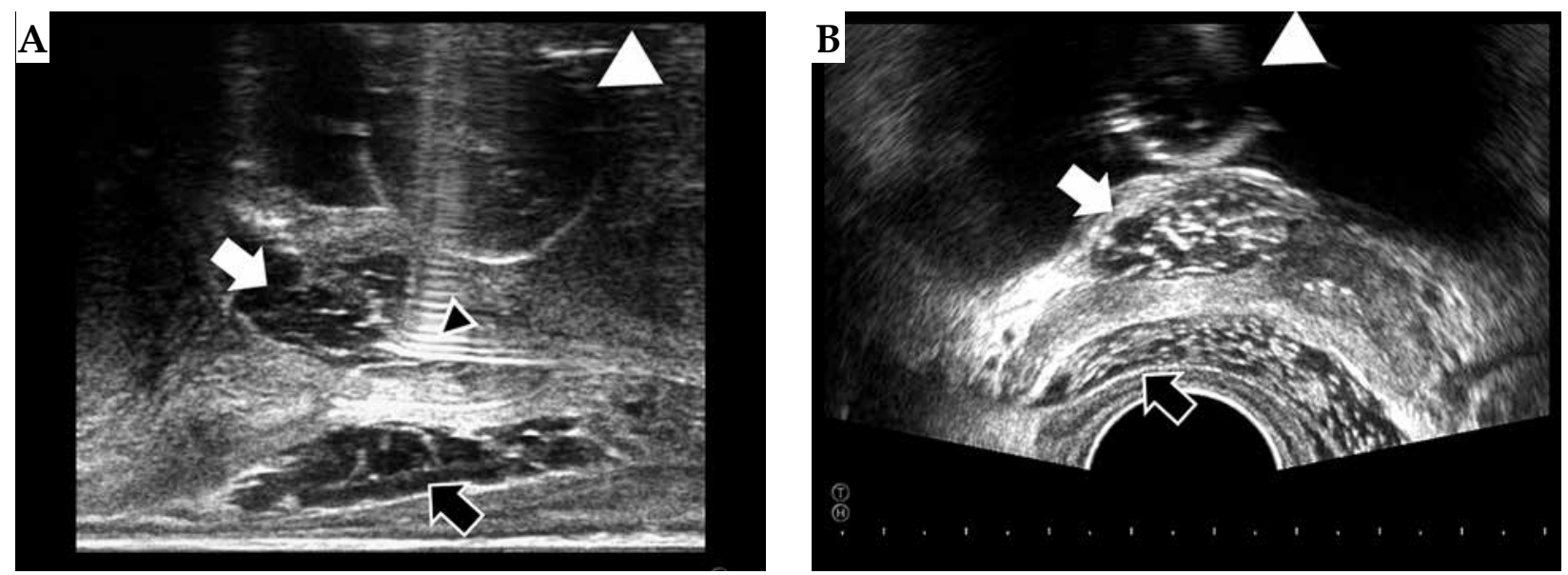

Fig. 1. Trans-rectal ultrasonography (TRUS) of hyaluronic acid gel injection (HGI) in vesicovaginal septum (VVS) and rectovaginal septum (RVS). A) Sagittal view of the TRUS. White and black arrow indicates hyaluronic acid gel inserted in the VVS and the RVS, respectively. Small arrow head indicates needle inserted in VVS. Large white arrow head indicates bladder balloon. B) Axial view of the TRUS. White and black arrow indicates hyaluronic acid gel inserted in the VVS and the RVS, respectively. The vagina was sandwiched by hyaluronic acid gel. Large white arrow head indicates bladder balloon 
Hyaluronic acid gel injection in the VVS was performed with a $19 \mathrm{G}$ needle (disposable ultrasonography compatible puncture needle, Create Medic, Co., LTD., Kanagawa, Japan) under the transrectal ultrasound (TRUS) guidance through the anterior wall of the vagina. Patients with anterior vaginal wall or bladder wall invasion were excluded because of potentially tumor cell dissemination or under dose of bladder wall. In order to avoid urethral injury, the needle was inserted via anterior wall of the vagina beyond the Foley catheter balloon protuberance, which was fixed to the bladder neck. In order to avoid laterally situated ureter injury, the needle was kept in the midline. Five vials of sunenyl (Chugai Phar- maceutical Co., Tokyo, Japan) diluted with $5 \mathrm{ml}$ of saline and 2-4 $\mathrm{ml}$ of contrast enhancement agent (oiparomin 370; Fuji Pharmaceutical Company, Toyama, Japan) were injected in the VVS and in the RVS (Figure 1). With this technique, the vagina could be sandwiched anteriorly and posteriorly by hyaluronic acid gel. Unlike the rectal side, only $5-10 \mathrm{ml}$ of suvenyl solution could be injected in the VVS due to comparatively limited space. In addition to the HGI in the VVS, usual gauze packing above and below the ovoids was performed. Figure 2 shows an example of axial and sagittal CT images of a patient with and without HGI in the VVS with BT applicators in situ. Figure 3 shows the BT dose distribution for the same pa-
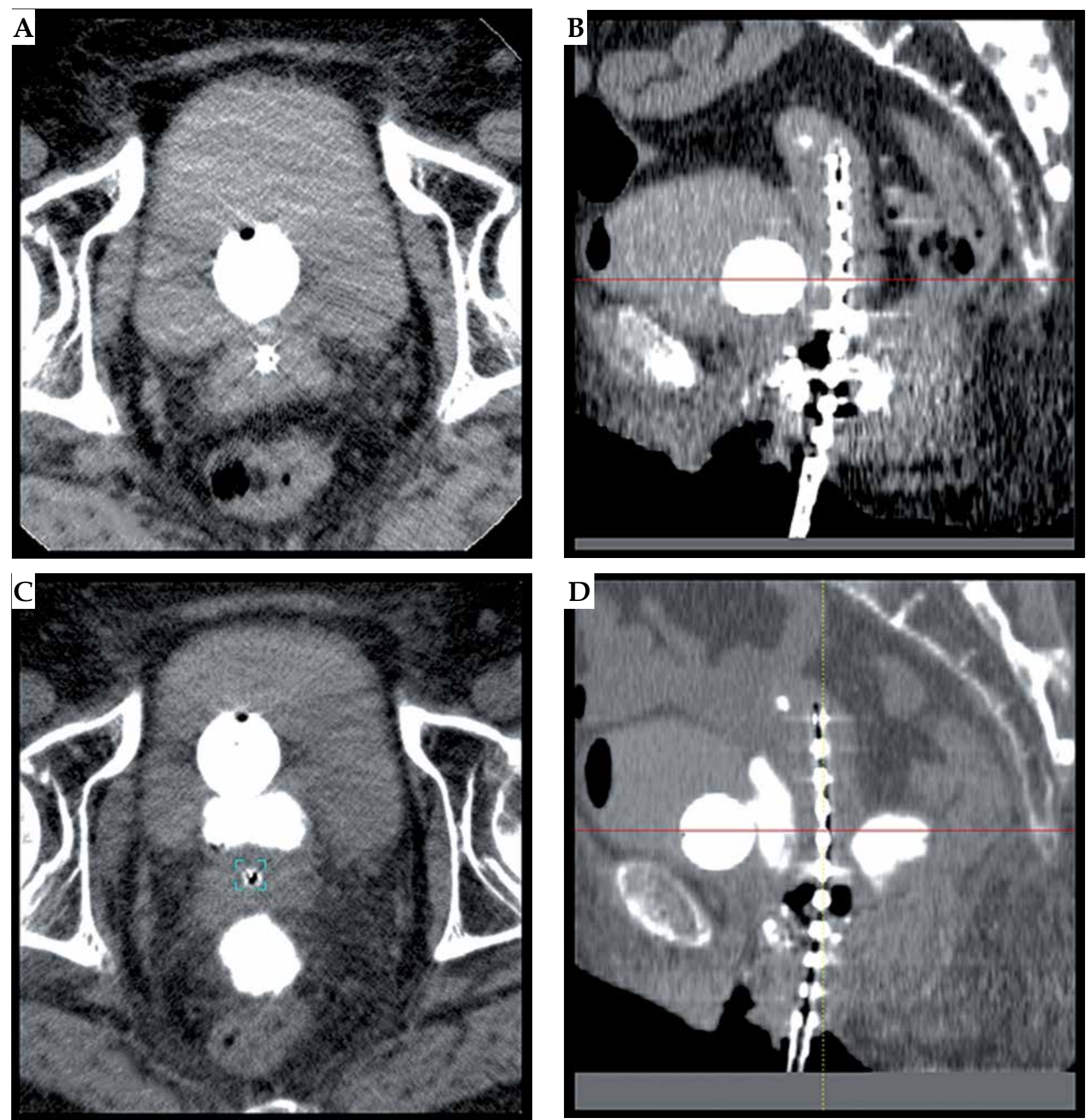

Fig. 2. A, B) Axial and sagittal CT image without HGI with applicators in place. C, D) Axial and sagittal CT image of the same patient in another brachytherapy session with HGI in the VVS and the RVS with applicators in place 

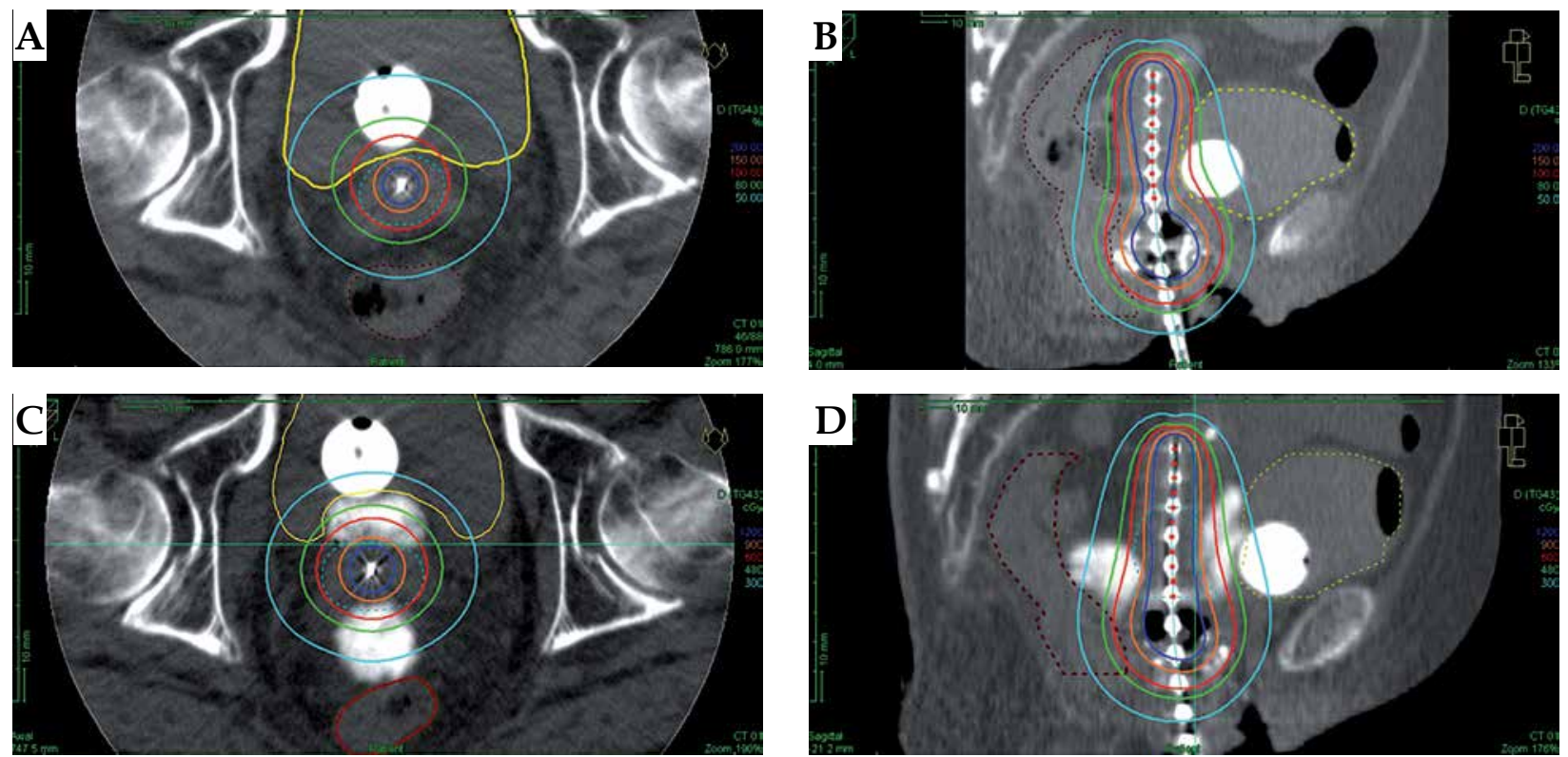

Fig. 3. Distribution of the intra-cavitary brachytherapy for uterine cervical cancer patient. The bladder was contoured with yellow line and 100\% isodose line (6 Gy) was indicated by red line. A, B) Axial and sagittal CT image without HGI with applicators in place. C, D) Axial and sagittal CT image of the same patient in another brachytherapy session with HGI in the VVS and the RVS with applicators in place. While the red line invaded the bladder in A and B, the red line existed outside of the bladder in the $\mathrm{C}$ and $\mathrm{D}$

tient with and without HGI in the VVS. The bladder is contoured yellow, and the 6 Gy isodose $(100 \%$ isodose, i.e. prescription dose) is represented by the red line. In Figure $3 \mathrm{~A}$ and $\mathrm{B}$, the $100 \%$ isodose is covering bladder tissue, while in Figure $3 C$ and D, the $100 \%$ isodose is not affecting the bladder due to HGI.

Anatomy-oriented dose optimization was performed in order to cover the HR-CTV by the 6 Gy isodose, while OAR dose constraints should be kept within guideline recommendations [9,11]. For obtaining the combined dose of EBRT + BT, the equivalent dose in 2 Gy fractions (EQD2) [9] was calculated according to LQ model [20] using the formula:

$$
\mathrm{EQD}_{2}=N d\{1+\mathrm{d} /(\alpha / \beta)\} /\{1+2 /(\alpha / \beta)\},
$$

in which $N$ represents the number of fractions and $d$ the dose per fraction. The $\alpha / \beta$ ratio was assumed $10 \mathrm{~Gy}$ and 3 Gy for tumor and normal tissues, respectively [21,22]. Because central parts of the pelvis do not receive irradiation during CS, only the whole pelvic EBRT doses were used for $\mathrm{EQD}_{2}$ calculation. Dose constraints for OARs are as follows: rectum $\mathrm{D}_{2 \mathrm{cc}}<75 \mathrm{~Gy}\left(\mathrm{EQD}_{2}\right)$, bladder $\mathrm{D}_{2 \mathrm{cc}}$ $<90$ Gy $\left(\mathrm{EQD}_{2}\right)$.

In order to enable a straightforward dosimetric comparison, all patients receiving multiple BT fractions both with and without HGI in the VVS through their course of RT were selected. Dosimetric parameters such as blad$\operatorname{der} \mathrm{D}_{2 \mathrm{cc}}$ (most exposed $2 \mathrm{cc}$ of bladder dose), HR-CTV $\mathrm{D}_{90}$ (minimum dose covering $90 \%$ of the HR-CTV), and rectum $\mathrm{D}_{2 \mathrm{cc}}$ (most exposed $2 \mathrm{cc}$ of rectum dose) were compared. Each patient had dose-volume-histogram (DVH) parameters for each BT fraction with or without HGI in the VVS. Because the number of BT fractions with or with- out HGI in the VVS differed between patients, for each patient, the dose parameters of each BT fraction were averaged and categorized in groups with and without HGI in the VVS to create one representative dose for a specific patient in each group. In two patients, overall two BT sessions were excluded from analysis due to a prescription dose $<6$ Gy. Paired $t$-test was performed to compare the mean value difference of each dosimetric parameter. $P$ value $<0.05$ was considered to be statistically significant.

Written informed consent was obtained from all patients. This retrospective study was approved by the institutional review board of the National Cancer Center Hospital (approval number 2017-091) according to the ethical standards laid down in the Declaration of Helsinki.

\section{Results}

The cumulative $\mathrm{EQD}_{2}$ for HR-CTV, rectum $\mathrm{D}_{2 \mathrm{cc}}$ and bladder $\mathrm{D}_{2 \mathrm{cc}}$ for the 9 patients are summarized in Table 2 . The median number of BT fractions was four (range, 2-5). Four, one, three, and one patient received HGI in the VVP once, two, three, and four times in their course of RT, respectively. Table 3 represents the DVH parameters comparison normalized at $6 \mathrm{~Gy}$ in single fraction between BT fractions with and without HGI in the VVS. Figure 4 demonstrates overview of plots of individual bladder $D_{2 c c}$ value for each of the 9 patients. Normalization was performed because the number of BT fractions differed according to the tumor response, and 6 Gy was our standard single prescription dose. Two patients who received under 6 Gy per fraction of BT as a final boost BT session for the residual tumor and these two last sessions were excluded from the analysis. While no statistical difference could be detected for rectal dose reduction, bladder dose 
Table 3. DVH parameters comparison between brachytherapy sessions with and without hyaluronic acid gel injection (HGI) in the vesicovaginal septum (VVS)

\begin{tabular}{|c|c|c|c|}
\hline & With HGI in the VVS & Without HGI in the VVS & $p$ value \\
\hline Median HR-CTV D 90 (cGy) & 713 (range, 671-825, 1SD = 57.3) & 706 (range, 612-751, 1SD = 42.8) & 0.085 \\
\hline Median HR-CTV $D_{90}\left(G y E Q D_{2}\right)$ & 10.7 (range, 9.3-14.4, 1SD = 1.21) & 10.2 (range, 8.4-11.6, 1SD = 0.85) & 0.084 \\
\hline Median bladder $\mathrm{D}_{2 c c}$ (cGy) & $449($ range, $416-566,1 S D=66.1)$ & $569($ range, $449-647,1 S D=59.5)$ & $0.033^{*}$ \\
\hline Median bladder $D_{2 c c}\left(G y E Q D_{2}\right)$ & $7.8($ range, 5.3-11.0, 1SD = 1.71) & 9.7 (range, 5.2-12.3, $1 \mathrm{SD}=1.60$ ) & $0.033^{*}$ \\
\hline Median mean bladder dose (cGy) & 191 (range, $162-254,1 S D=30.2$ ) & 210 (range, $174-290,1 S D=37.3)$ & 0.055 \\
\hline Median mean bladder dose $\left(\mathrm{Gy} E Q D_{2}\right)$ & 1.9 (range, $1.2-2.8,1 \mathrm{SD}=0.42)$ & 2.1 (range, $1.7-3.4,1 \mathrm{SD}=0.56)$ & 0.054 \\
\hline Median rectum $\mathrm{D}_{2 c c}(\mathrm{cGy})$ & 400 (range, 309-497, 1SD = 69.1) & 423 (range, 290-564, 1SD = 91.9) & 0.755 \\
\hline Median rectum $\mathrm{D}_{2 c \mathrm{c}}\left(\mathrm{Gy} E Q \mathrm{D}_{2}\right)$ & $6.0($ range, $3.6-8.4,1 S D=1.56)$ & $6.5($ range, $3.4-10.2,1 S D=2.14)$ & 0.674 \\
\hline
\end{tabular}

$H G I$ - hyaluronic acid gel injection, VVS - vesicovaginal septum, HR-CTV - high-risk clinical target volume, SD - standard deviation, EQD2 - the equivalent dose in 2 Gy fractions

was significantly less in the group with HGI compared to that without (median bladder $\mathrm{D}_{2 \mathrm{cc}} 449$ cGy [range, 416$566,1 \mathrm{SD}=66.1$ ] vs. 569 cGy [range, 449-647, $1 \mathrm{SD}=59.5$ ], $p=0.033$ ), with no compromising of the target coverage. On the contrary, although it did not reach statistically significance, there was a trend towards better HR-CTV $\mathrm{D}_{90}$ in the group with HGI compared to that without HGI in the VVS (713 cGy vs. 706 cGy, $p=0.085$ ).

No severe bleeding, hematuria, bladder wall injury, or urethral injury requiring hospitalization was experienced in association with HGI in the VVS, while mild bleeding from the anterior wall of the vagina or penetration of bladder base that did not required need/prolongation of the hospitalization was observed.

\section{Discussion}

Image-guided BT (IGBT) is an integral component of primary RT for uterine cervical cancer contributing to improved pelvic control and survival compared to chemoradiation therapy (CRT) by sole EBRT $[6,7,23]$. Against this background, quality of life issues has gained increasing importance for treatment decision making, with BT having proved next to high efficacy also low morbidity [23]. This is because HDR-BT meets the objective of conformal dose escalation optimally by exploiting the radiobiological advantage of extreme hypofractionation, while ensuring superior 3D dosimetry. At this point, the versatility of intratarget dose modulation inherent to afterloading BT enables dosimetric optimization, which cannot be reproduced even with the most conformal external beam techniques [24], thus enhancing the therapeutic ratio by decreasing higher grade OARs toxicity, such as rectal ulcer and rectovaginal or vesicovaginal fistula [25].

To this effect, next to efforts improving conformity by IGBT, also vaginal gauze packing or rectal retractor devices were used to create additional space between target volume and OARs. However, in various clinical settings, it still remains challenging to fulfill the dose constrains recommended by the Groupe European de Curietherapie-European society for radiation therapy (GEC-ESTRO) [9] and the American Brachytherapy Society (ABS) [11]

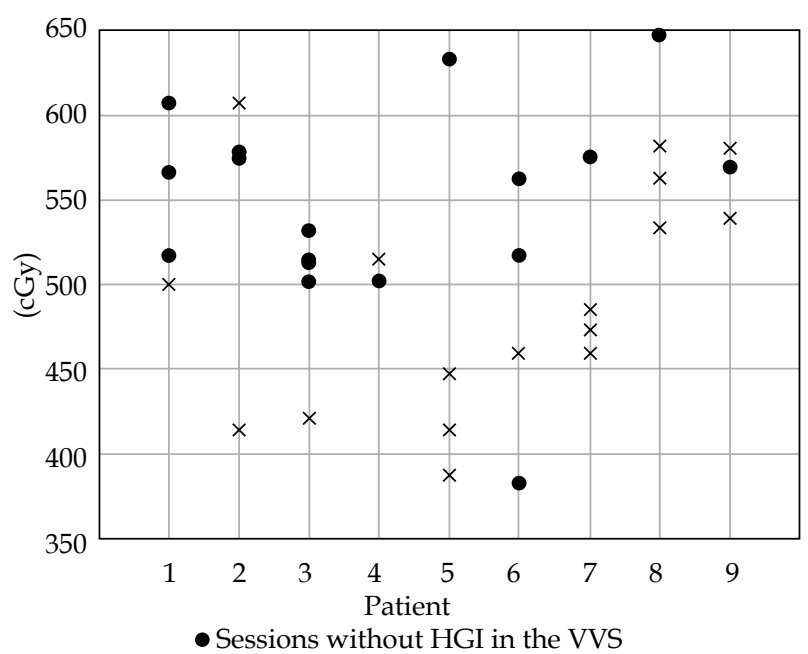

$\times$ Sessions with HGI in the VVS

Fig. 4. Overview of plots of individual bladder $\mathrm{D}_{2 \mathrm{cc}}$ value for each 9 patients. Black cross and circle represents brachytherapy sessions with and without HGI in the VVS, respectively

for the definitive RT treatment of locally advanced carcinoma of the cervix. Some researchers tried to find optimal bladder filling volume, which most likely reduce dose of OARs (bladder, rectum and sigmoid colon) in image-guided BT for cervical cancer [26]. At this point, HGI has shown to be a further aid to create safely space between OARs and tumor volume both in BT $[12,13,14,15]$ and in EBRT $[16,17]$. In this study, we used HGI in the VVS in an attempt to reduce bladder dose effectively and demonstrated that this was safely possible. Minor bleeding from vaginal wall or bladder could be easily managed by simple vaginal packing or bladder irrigation. Moreover, our findings support that HGI not only reduces the dose to OARs but might also contribute to safe target dose escalation. Damato et al. reported HGI in the VVS and RVS using cadaver [27] and reported that HGI could reduce the OARs dose less than with only usual gauze packing. The same group also reported usage of HGI 
to create space between vaginal cuff and bowel in the post-operative case [28]. To the best of our knowledge, this is the first report on the feasibility and dosimetric superiority of HGI in the VVS for bladder sparing in uterine cervical cancer BT for actual patients. One vial of sunenyl costs only 725 Japanese yen (5.66 Euro), therefore, it can be said that this procedure is quite economical.

There are several limitations in this study. This is a retrospective analysis with a limited number of patients. Moreover, this study only demonstrated dosimetric superiority of HGI in the VVS for bladder sparing. However, it is unclear if this dosimetric superiority will be translated into meaningful clinical benefits. Mazeron et al. reported in a study with median follow-up of 35 months that bladder $D_{2 c c}$ for $10 \%$ grade 2 or greater and grade 3 or greater were $55.4 \mathrm{~Gy}$ and $85.3 \mathrm{~Gy}$, respectively [29], which suggested that even small reduction of bladder dose would translate into later toxicity-free survival. Therefore, further clinical research is needed to find its long-term efficacy. It is easy to imagine that patients with bladder invasion should not receive HGI in the VVS because this would prohibit the delivery of an adequate dose to the bladder neck. It might also be true that in the era of IGABT, most patients do not experience severe late side effect after definitive CRT without HGI in the VVS. However, in keeping with the belief that "the better is the enemy of the good", more research is justified in order to fully explore the potential HGI in the VVS from a clinical point of view.

\section{Conclusions}

HGI in the VVS can be performed safely and can effectively reduce the bladder dose in BT for uterine cervical cancer patients.

\section{Acknowledgements}

This study was partially supported by the Japan Agency for Medical Research and Development, AMED, the National Cancer Center Research and Development Fund (26-A-18 and 26-A-28).

\section{Disclosure}

Authors report no conflict of interest.

\section{References}

1. Tod M, Meredith WJ. Treatment of cancer of the cervix uteri, a revised Manchester method. Br J Radiol 1953; 26: 252-257.

2. Whitney CW, Sause W, Bundy BN et al. Randomized comparison of fluorouracil plus cisplatin versus hydroxyurea as an adjunct to radiation therapy in stage IIB-IVA carcinoma of the cervix with negative para-aortic lymph nodes: a Gynecologic Oncology Group and Southwest Oncology Group study. J Clin Oncol 1999; 17: 1339-1348.

3. Rose PG, Bundy BN, Watkins EB et al. Concurrent cisplatin-based radiotherapy and chemotherapy for locally advanced cervical cancer. N Engl J Med 1999; 340: 1144-1153.

4. Morris M, Eifel PJ, Lu J et al. Pelvic radiation with concurrent chemotherapy compared with pelvic and para-aortic radiation for high-risk cervical cancer. N Engl J Med 1999; 340: 1137-1143.
5. Landoni F, Maneo A, Colombo A et al. Randomised study of radical surgery versus radiotherapy for stage Ib-IIa cervical cancer. Lancet 1997; 350: 535-540.

6. Han K, Milosevic M, Fyles A et al. Trends in the utilization of brachytherapy in cervical cancer in the United States. Int J Radiat Oncol Biol Phys 2013; 87: 111-119.

7. Mayadev J, Klapheke A, Yashar C et al. Underutilization of brachytherapy and disparities in survival for patients with cervical cancer in California. Gynecol Oncol 2018; 150: 73-78.

8. Georg P, Potter R, Georg D et al. Dose effect relationship for late side effects of the rectum and urinary bladder in magnetic resonance image-guided adaptive cervix cancer brachytherapy. Int J Radiat Oncol Biol Phys 2012; 82: 653-657.

9. Pötter R, Haie-Meder C, Van Limbergen E et al. Recommendations from gynaecological (GYN) GEC ESTRO working group (II): concepts and terms in 3D image-based treatment planning in cervix cancer brachytherapy-3D dose volume parameters and aspects of 3D image-based anatomy, radiation physics, radiobiology. Radiother Oncol 2006; 78: 67-77.

10. Mazeron R, Fokdal LU, Kirchheiner K et al. Dose-volume effect relationships for late rectal morbidity in patients treated with chemoradiation and MRI-guided adaptive brachytherapy for locally advanced cervical cancer: Results from the prospective multicenter EMBRACE study. Radiother Oncol 2016; 120: 412-419.

11. Viswanathan AN, Beriwal S, De Los Santos JF et al. American Brachytherapy Society consensus guidelines for locally advanced carcinoma of the cervix. Part II: high-dose-rate brachytherapy. Brachytherapy 2012; 11: 47-52.

12. Kishi K, Iida T, Ojima $T$ et al. Esophageal gel-shifting technique facilitating eradicative boost or reirradiation to upper mediastinal targets of recurrent nerve lymph node without damaging esophagus. J Radiat Res 2013; 54: 748-754.

13. Kishi K, Mabuchi Y, Sonomura T et al. Eradicative brachytherapy with hyaluronate gel injection into pararectal space in treatment of bulky vaginal stump recurrence of uterine cancer. J Radiat Res 2012; 53: 601-607.

14. Kishi K, Sonomura T, Shirai $\mathrm{S}$ et al. Brachytherapy reirradiation with hyaluronate gel injection of paraaortic lymphnode metastasis of pancreatic cancer: paravertebral approach a technical report with a case. J Radiat Res 2011; 52: 840-844.

15. Kishi K, Sonomura T, Shirai S et al. Critical organ preservation in reirradiation brachytherapy by injectable spacer. Int J Radiat Oncol Biol Phys 2009; 75: 587-594.

16. Mariados N, Sylvester J, Shah D et al. Hydrogel spacer prospective multicenter randomized controlled pivotal trial: dosimetric and clinical effects of perirectal spacer application in men undergoing prostate image guided intensity modulated radiation therapy. Int J Radiat Oncol Biol Phys 2015; 92: 971-977.

17. Hamstra DA, Mariados N, Sylvester J et al. Continued benefit to rectal separation for prostate radiation therapy: final results of a phase III trial. Int J Radiat Oncol Biol Phys 2017; 97: 976-985.

18. Murakami N, Kasamatsu T, Wakita A et al. CT based three dimensional dose-volume evaluations for high-dose rate intracavitary brachytherapy for cervical cancer. BMC Cancer 2014; 14: 447.

19. Kuroda Y, Murakami N, Morota M et al. Impact of concurrent chemotherapy on definitive radiotherapy for women with FIGO IIIb cervical cancer. J Radiat Res 2012; 53: 588-593.

20. Dale RG. The application of the linear-quadratic dose-effect equation to fractionated and protracted radiotherapy. $\mathrm{Br}$ J Radiol 1985; 58: 515-528.

21. Stuschke M, Thames HD. Fractionation sensitivities and dose-control relations of head and neck carcinomas: analysis of the randomized hyperfractionation trials. Radiother Oncol 1999; 51: 113-121. 
22. Terry NH, Denekamp J. RBE values and repair for colo-rectal injury after caesium 137 gamma-ray and neutron irradiation. II. Fractionation up to ten doses. Br J Radiol 1984; 57: 617-629.

23. Sturdza A, Potter R, Fokdal LU et al. Image-guided brachytherapy in locally advanced cervical cancer: Improved pelvic control and survival in RetroEMBRACE, a multicenter cohort study. Radiother Oncol 2016; 120: 428-433.

24. Otahal B, Dolezel M, Cvek J et al. Dosimetric comparison of MRI-based HDR brachytherapy and stereotactic radiotherapy in patients with advanced cervical cancer: A virtual brachytherapy study. Rep Pract Oncol Radiother 2014; 19: 399404.

25. Dang YZ, Li P, Li JP et al. The efficacy and late toxicities of computed tomography-based brachytherapy with intracavitary and interstitial technique in advanced cervical cancer. J Cancer 2018; 9: 1635-1641.

26. Sharma AD, Poddar J, Suryanarayan KU et al. Dosimetric analysis of the effects of the bladder volume on organs at risk (OAR) in high-dose-rate intracavitary brachytherapy in carcinoma cervix - an institutional study. J Contemp Brachytherapy 2018; 10: 26-31.

27. Damato AL, Kassick M, Viswanathan AN. Rectum and bladder spacing in cervical cancer brachytherapy using a novel injectable hydrogel compound. Brachytherapy 2017; 16: 949955.

28. Viswanathan AN, Damoto AL, Nguyen PL. Novel use of a hydrogel spacer permits reirradiation in otherwise incurable recurrent gynecologic cancers. J Clin Oncol 2013; 31: e446-447.

29. Mazeron R, Maroun P, Castelnau-Marchand P et al. Pulseddose rate image-guided adaptive brachytherapy in cervical cancer: Dose-volume effect relationships for the rectum and bladder. Radiother Oncol 2015; 116: 226-232. 